\title{
How to Keep Bad Papers Out of Conferences (with Minimum Reviewer Effort)
}

\author{
Jonathan Anderson, Frank Stajano, and Robert N.M. Watson \\ University of Cambridge Computer Laboratory, Cambridge, UK \\ \{firstname.lastname\}@cl.cam.ac.uk
}

\begin{abstract}
Reviewing conference submissions is both labour-intensive and diffuse. A lack of focus leads to reviewers spending much of their scarce time on papers which will not be accepted, which can prevent them from identifying several classes of problems with papers that will be. We identify opportunities for automation in the review process and propose protocols which allow human reviewers to better focus their limited time and attention, making it easier to select only the best "genetic" material to incorporate into their conference's "DNA." Some of the protocols that we propose are difficult to "game" without uneconomic investment on the part of the attacker, and successfully attacking others requires attackers to provide a positive social benefit to the wider research community.
\end{abstract}

\section{Introduction}

One view of a Program Committee's role is the defence of sacrosanct publication venues - conferences, journals, workshops - from invading $\mathrm{PhD}$ students1. Committee members wish to admit worthy researchers as fellow-guardians of the state of the art. Unfortunately, most of their limited and valuable time is spent repelling hordes of unworthy submitters, trying to get published with minimal effort and literacy in the ancient lore of the discipline. A second view casts the Program Committee as an individual member of a species, attempting to select the mate that will most increase the genetic fitness of their offspring. In this case, the committee's goal is to encourage the survival of the discipline by populating it with strong researchers-promoting the Darwinian fitness of its publications [4] and to stave off monoculture through the continual integration of fresh new problems and ideas.

Figure 1 shows the trend of some research communities built around publication venues - both security-centric and otherwise - to cite their own work more and more over time, to the exclusion of "outside" research 2 . Obviously, work

\footnotetext{
${ }^{1}$ Readers should note that, at the time of writing, two of this paper's authors were $\mathrm{PhD}$ students, tongues firmly placed in their cheeks.

2 These graphs have been generated from data supplied by arnetminer.org [67], itself based on DBLP. Despite a manual cleanup by the authors of this paper, there are some inconsistencies (e.g. citations of a technical report from the previous year turn up as citations of the journal article version, from two years in the future).
} 


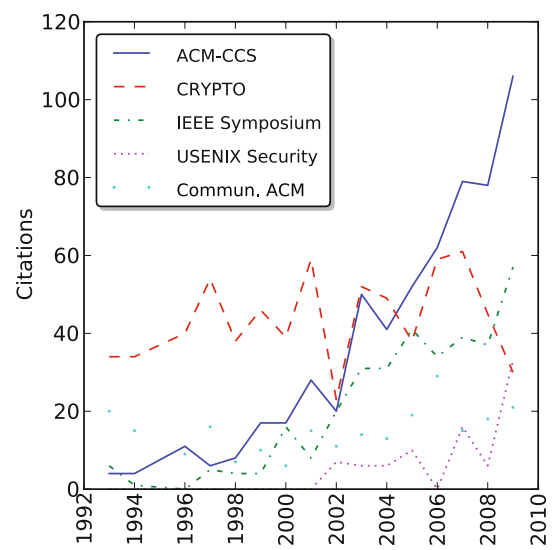

(a) ACM-CCS

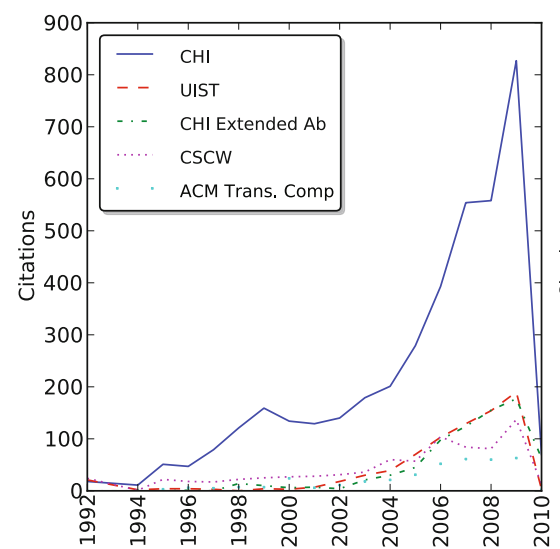

(c) $\mathrm{CHI}$

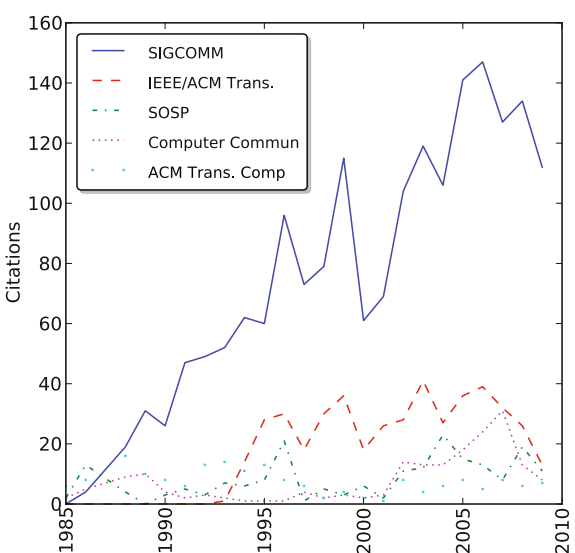

(b) SIGCOMM

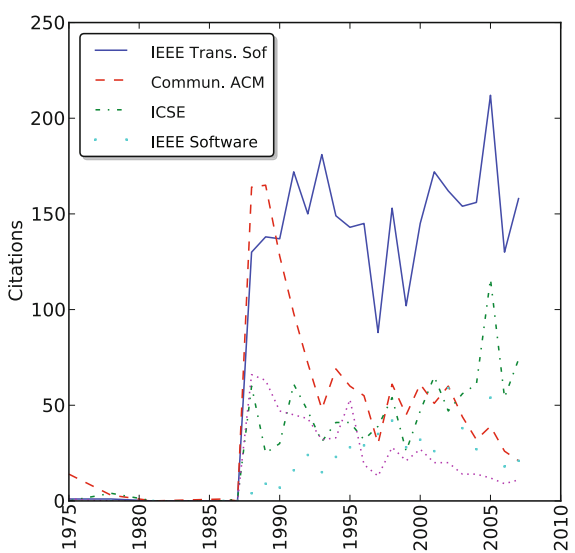

(d) IEEE Trans. on Soft. Eng.

Fig. 1. Frequency with which papers at four conferences cite work from various venues

published at a particular conference will tend to cite other work from the same community - papers accepted by WiSec will be focussed on wireless security, and will thus tend to cite previous WiSec papers - but it is possible that some conferences may be growing quite introspective, delving deeper and deeper into the depths of known problems without continual exposure to the new "genetic material" (problems and ideas) that is essential for the health of any community. It is, of course, important for research communities to optimise existing solutions to existing problems, but if authors only submit "the kind of paper that always gets in," if new ideas and new problems are never explored, the overall health of the community may suffer. Offspring of such an unhealthy community are unlikely to be selected by discerning partners - in academic terms, their work will not be cited outside their own community. 
In this work, we describe threats to the health of academic disciplines, including unscrupulous authors faking genetic fitness and established communities selecting only their own academic offspring (Section 2). We propose mitigating these problems via the judicious application of automation: reviewers can apply statistical techniques to measurable things in order to focus human attention on the subjective quality of conference submissions. We can remove some labourious work from reviewers' shoulders (Section 3.1): we can force attackers to step up their game, ultimately writing better papers or, if attempting to game the system, provide useful benefits to the wider research community (Section 3.2). We can look for authors attempting to socially engineer themselves into acceptance (Section 3.3), and we can promote diversity of ideas within conferences, reducing monocultures and increasing the health of the community (Section 3.4).

\section{Threat Model}

In our threat model, the attacker is the unscrupulous author of a submission. These authors may be seen as attempting to gain entry to the "ivory tower" of an academic discipline without first proving their worth through strong research. Alternatively, we may view the attacker as an individual attempting to "mate" with a conference without demonstrating "genetic" fitness or the likely fitness of potential offspring (research which successful publication might inspire).

The attacker can attempt to frustrate the proper functioning of the Program Committee by abusing citations or coasting on reputation. Citations are meant to be a signal that an author has read and understood the work of others. Unscrupulous authors, however, can tailor them to the program committee at little cost - citing what they've written, citing what they like, citing what will make them think the authors are very well versed in the literature, all without actually reading and understanding the work they reference. Established authors, who have previously published at a conference, can also send the kind of signals that they know will be well-received, whereas proving one's fitness to a new research community may require truly superior research in order to overcome that community's institutional resistance to change. Such attacks are successful today in part because it is much more time-consuming for the reviewer to verify a citation than for the attacker to insert one, and the reviewer has many citations which she might choose to review.

Furthermore, the attacker can submit "the kind of paper that always gets in to this conference", which has the twin effects of increasing the chances of unworthy research and reinforcing a vicious cycle of monoculture begetting monoculture. Such a cycle might, in the end, lead to the stagnation of the discipline.

Finally, we recognise that attackers, in current systems, can effectively carry out a Denial of Service attack on reviewers, distracting them with the necessity of refuting incorrect statements and correcting poor writing. This "busy work" may keep them from arguably more important tasks, such as shepherding good papers or detecting subtle flaws (such as incorrect proofs) in bad ones. 


\title{
3 Mechanical Assistance
}

Reviewers' time is a scarce resource, which a conference review process should spend carefully. Assuming that semantically-meaningful content of submissions can be reliably extracted (e.g. submission requires latex sources as well as PDF files, or PDF-to-text is reliable), thre are several ways in which mechanical assistance can be provided to program committees. Such assistance will allow reviewers to focus their scarce attention on the aspects of the review process which cannot be automated - evaluating the quality of ideas and research.

\subsection{Clustering Submissions}

Today, some conferences ask authors to provide keywords that describe their work, as in Figure 2. Where employed, this scheme makes it easier for review tasks to be distributed among reviewers. Nonetheless, significant human effort is involved in sifting through e.g. all of the abstracts tagged "committment schemes." We propose that mechanical analysis of submissions' reference lists could augment this process.

We presume that submissions can be usefully classified by their citations: papers that cite similar work are likely to be about similar topics. Indeed, it has long been known that it is possible to mechanically classify academic publications into sub-fields via the citation graph without any special knowledge of the content of the literature [2]. Rather than asking reviewers to review a set of abstracts, then, reviewers might be asked to rank their interest in existing

Review abstracts tagged with keywords:

\author{
$\varpi$ Commitment Schemes \\ $\square$ Mobile Phones \\ ØOperating Systems
}

\section{Submission \#3}

Lorem ipsum dolor sit amet, consectetur adipiscing elit. Ut ac tempor metus. Praesent vitae urna dui. Cras sed nulla diam. Mauris facilisis consectetur odio, fermentum dignissim lacus cursus nec. Pellentesque massa enim, ultricies congue luctus sed, mattis eget orci. Vestibulum ante ipsum primis in faucibus orci luctus et ultrices posuere cubilia Curae; Curabitur vel felis lorem. Phasellus justo nisi, eleifend ac vulputate quis, dapibus ut urna.

$\square$ I want to review this paper

\section{Submission \#6}

Quisque condimentum turpis ac enim vulputate dictum. Curabitur sem ligula, lobortis sit amet euismod a, pretium et tellus. Suspendisse tempor elit sed nulla molestie ut faucibus massa varius. Morbi purus augue, adipiscing non porta eu, accumsan in nibh. Proin tempus arcu eget est viverra in tempor metus dignissim. Aliquam erat volutpat. Integer faucibus dictum tellus, ac faucibus nibh pellentesque non.

\section{$\searrow$ I want to review this paper}

\section{Submission \#7}

Nulla iaculis faucibus purus sit amet consequat. Etiam condimentum eros vel sapien tristique posuere. Proin iaculis tempor vestibulum. Praesent ut sem sed felis accumsan interdum. Praesent adipiscing libero eu nulla fringilla blandit. Sed ut tortor quis nunc congue tempor a eget augue.

$\checkmark$ I want to review this paper

Fig. 2. Selecting papers to review via author-supplied keywords and abstracts 
classic papers which have been calculated to convey the most information about clustering via e.g. principal components analysis [3]. Such a list of papers, shown in Figure 3. might even be pre-populated with suggested selections based on the reviewer's authorship and citation records, greatly reducing the amount of work, but still providing reviewers with the opportunity to express disinterest in work that they did in the past but have since lost interest in.

Which of the following are you most familiar with:

"A new problem", Alice et al., Security Protocols Workshop, 1995.

"First crack at Alice's problem", Bob and Sam, Security Protocols Workshop, 2005.

$\checkmark$ "A better solution than Bob and Sam's", Eve and Moriarty, Security Protocols Workshop, 2009.

Fig. 3. Selecting papers to review via citation clusters

Furthermore, the results of citation-based classification can be compared with author-supplied keywords. Submissions whose keywords do not match the automatic classification can then be flagged as "interesting", either because the authors are using keywords in a clueless manner, or because their research defies the existing keyword classification scheme. Which of the two is true must be determined by a human reviewer, but checking a small portion of the total submissions for "interestingness" could be a much better use of reviewers' time than trawling through vast oceans of submitted abstracts.

\subsection{Signalling That Authors Possess What They Cite}

A paper's citations are like a bird's plumage, enhancing the chances of the subject to be selected by a discriminating audience with a large field of suitors. Given the stakes, there are obvious motivations to exaggerate one's citations: it makes the authors appear literate, lending credibility to the submission; it may flatter members of the Program Committee; it may be seen as a prerequisite to working in the field ("you can't publish here unless you cite Smith's seminal work on Public Key Widgets"). Such behaviour dilutes the quality of the conference in the long run, however: it fills reference lists with meaningless data, reducing the amount of information per page of proceedings.

In order to discourage such behaviour, we propose an information signalling protocol. This protocol requires authors to signal that, at minimum, they have gone through the trouble of finding and looking at (though not necessarily reading thoroughly) everything that they cite. The protocol requires little reviewer effort and communications overhead, and can even be verified after publication by any third party who can read the bibliography. The protocol is in-band, relying on the annotation of citations, but authors cannot simply replay annotated citations from other papers.

Such a signalling protocol cannot guarantee that an author has, in fact, read and understood everything they cite. If we assume, however, that the "energy gap" between opening a PDF and actually skimming its conclusion is not large 
enough to overcome PhD students' genuine interest in learning, this should be a very useful signal.

In the reference list at the end of a submission, we require authors to annotate each citation with a single word within square brackets. This word is taken from the referenced document, a response to a challenge which is public but unique. This challenge, $c_{i}$ (the challenge associated with reference $i$ ), is given by:

$$
c_{i}=\left(n_{i} \bmod P, n_{i} \bmod N\right) \mid n_{i}=h\left(h_{m}(s)\left|h_{m}(i)\right| n_{c}\right)
$$

where $n_{i}$ is a nonce specific to a particular reference in a particular submission, $P$ is the number of pages in the referenced document and $N$ is a number small enough to be easily counted by humans (e.g. less than 50$) \cdot h_{m}(x)$, the "metadata hash" of paper $x$, is defined as:

$$
h_{m}(p)=h\left(n_{c}|\operatorname{authors}(p)| \operatorname{conference}(p) \mid \operatorname{year}(p)\right)
$$

where $n_{c}$ is a nonce generated by the conference (perhaps the filename of a supplied LATEXclass), authors $(p)$ is a comma-separated list of last names of the paper's authors (in the same order as on the paper itself), etc. Including $h_{m}(s)$, the metadata hash of the submission, ensures that authors cannot collaborate in generating hashe 3 . Furthermore, if the authors have a partial list of words in the referenced document, the only way to change the challenge to a "favourable" value is by changing the author list - a high price to pay.

Verifying the responses associated with references should be a relatively loweffort task: most citations can be automatically examined, assuming the PC software has access to a large corpus of literature. Any "cache misses" (whether due to not having access to literature or poor PDF-to-text conversion) can be probabilistically flagged for human review, and the outcome will be a more complete corpus with better textual equivalents.

Finally, attackers could collude to build a large corpus of relevant literature with high-quality PDF-to-text conversion, but if they did, would it be such a bad thing? Surely such a corpus would be of benefit to the research community, although copyright holders may not be pleased 4 .

\subsection{Checking That Authors Have Read What They Cite}

A more difficult, and therefore more interesting, problem is checking that authors not only possess what they cite, but have read and understood it, critiquing it or allowing it to influence their own research. This is clearly the province of the experienced human reviewer, but mechanical assistance could be provided to reviewers to help them focus their energy where it would be most productive.

\footnotetext{
${ }^{3}$ That is, unless they collaborate to disseminate the relevant literature to researchers who haven't read it, which is surely a positive outcome!

${ }^{4}$ Of course, an wise publisher would use such a corpus to improve their own PDFs and citation graphs, again, a useful service to the research community.
} 
Identifying Usage. To fully combat the problem of "token citations", reviewers could look at every reference in every submission and ask themselves, "can I see the influence of the referenced work on the submission?" In the real world, such analysis is impossible due to time constraints. Software tools could help reviewers, however, by displaying every place a reference is cited, including a few additional lines of context. Such a tool, especially if employed primarily for "interesting" references (see below) could help reviewers make cursory inspections and quickly judge whether or not the cited work has an influence on the submission.

Identifying Outliers. One class of "interesting" references which are trivial to identify is that of statistical outliers. Figure 4 shows the increasing age of references, most of which are outliers, at the ACM Conference on Computer and Communications Security. These outliers date back to some of the seminal papers in computer security, and may be important references whose lessons have greatly influenced authors' thinking. Unfortunately, they may also be token citations which merely provide an air of historical literacy. Sorting the wheat from the chaff clearly must be done by a human; identifying which references particularly need to be sorted can be done more effectively by computer.

Identifying PC Citations. Another class of "interesting" references are those which have been written by Program Committee members. Clearly, PC members are more likely to be cited than the average author; if their work were not valuable to the field, they would not be on the committee! Nonetheless, authors

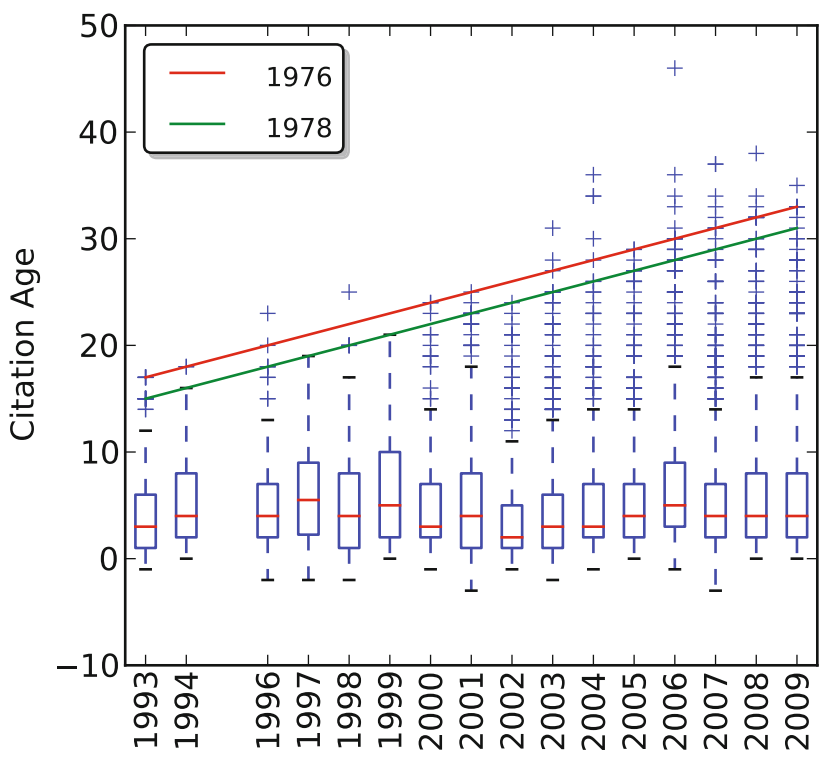

Fig. 4. Citation Age at ACM-CCS 
may be tempted to pad long strings of citations with PC members in an effort to flatter them or "pay their dues." Such citations are therefore more interesting than many others, and thus can be probabalistically flagged for human review.

\subsection{Encouraging Diversity}

Human reviewers can often sort submissions very quickly into three bins: "definitely accept", "definitely reject" and "maybe." Much effort then goes into deciding which of the "maybe" papers deserve to be accepted, even though the number of papers to be accepted may be a small fraction of the "maybe" category. In such cases, automation can help provide two properties that we consider useful: focussing the most reviewer effort on a small number of to-be-accepted submissions and encouraging diversity within the conference.

From biospheres to computer security, monoculture is often recognised as a systemic weakness [15], but as stated above, we may be able to observe a worrisome trend towards monoculture in Figure 1. In order to encourage diversity, then, PC software could treat submissions preferentially that cite substantially different work from the papers that will definitely be accepted - if the reviewers have already accepted six papers on the finer points of zero-knowledge proof, perhaps one paper about a new real-world problem would be a breath of fresh air, an injection of new "genetic material," even if the sixteen zero-knowledge proof papers in the "maybe" bin are slightly better written.

One interesting property of this scheme is that, even though it is a statisticalclassification-driven approach, it is very difficult to tactically adapt to: since its inputs are not "what got accepted last year", or even "what got submitted this year", but "what has been accepted this year", the kind of paper which will be most advantaged this year cannot be known until all of the first-pass reviews are in. The fact that social networks get an automated leg-up one year in no way implies that they will again next year: in fact, once they become a bandwagon, there will be pressure to get off the bandwagon and restore an interesting balance of work.

\section{Future Work}

We would like to conduct an experiment with a real program committee in which we ask each reviewer how much time they spent reviewing each submission, broken down into per-submission activities such as "reading up on things the submission cites", "convincing myself the idea works", "explaining why the idea doesn't work", "correcting grammar", etc 5

With more data, we would also like to explore the relationship between regular conference attendees and their publication records. Do authors who attend a conference every year tend to be more "introspective" than those who do not?

${ }^{5}$ Obviously, such data must be collected anonymously and could not be shared with the chair before aggregation, otherwise reviewers might experience some trepidation about honestly expressing how much or how little time they spend on each paper. 
Does attending a conference encourage others to cite your work, even if that work was not published at the conference in question? Only data can tell.

\section{Conclusion}

Through the judicious application of mechanical assistance, we believe that the conference submission review process can be made more efficient, focusing the limited time and energy resources of reviewers on those problems which can only be solved by humans. Furthermore, mechanical assistance could encourage good "genetic hygene" in conferences, leading to overall better health in the future.

\section{References}

1. Geer, D.J.: Monopoly considered harmful. IEEE Security \& Privacy 1(6), 14-17 (2003)

2. Kessler, M.: An experimental study of bibliographic coupling between technical papers. IEEE Transactions on Information Theory 9(1), 49-51 (1963)

3. Pearson, K.: On lines and planes of closest fit to systems of space. Philosophical Magazine 2(11), 559-572 (1901)

4. Simkin, M.V., Roychowdhury, V.P.: A Mathematical Theory of Citing. Journal of the American Society for Information Science and Technology 58(11) (2007)

5. Stamp, M.: Risks of monoculture. Communications of the ACM 47(3) ( March 2004)

6. Tang, J., Zhang, D., Yao, L.: Social Network Extraction of Academic Researchers. In: Seventh IEEE International Conference on Data Mining (ICDM), pp. 292-301 (2007)

7. Tang, J., Zhang, J., Yao, L., Li, J., Zhang, L., Su, Z.: ArnetMiner: extraction and mining of academic social networks. In: ACM SIGKDD International Conference on Knowledge Discovery and Data Mining (KDD). ACM (August 2008) 